\title{
Emergent CT Diagnosis of Right Ventricular Rupture Caused by Central Venous Catheter Injury
}

\author{
Sang Hoon Kim, ${ }^{1}$ Seung Min Yoo,,${ }^{2,}$ Hwa Yeon Lee, ${ }^{3}$ Gun Lee, ${ }^{4}$ Hyung Jong Kim, ${ }^{1}$ Minjung Shim, \\ Sung-ik Kim, ${ }^{1}$ In Song Song, ${ }^{5}$ Hye Rin Kim, ${ }^{2}$ and Charles S White ${ }^{6}$ \\ ${ }^{1}$ Department of Internal Medicine, CHA University College of Medicine, Bundang, Korea \\ ${ }^{2}$ Department of Diagnostic Radiology, CHA University College of Medicine, Bundang, Korea \\ ${ }^{3}$ Smile Radiologic Clinic, Seoul, Korea \\ ${ }^{4}$ Department of Cardiothoracic Surgery, CHA University College of Medicine, Bundang, Korea \\ ${ }^{5}$ Department of Diagnostic Radiology, Chunju Jesus Hospital, Chunju, Korea \\ ${ }^{6}$ Department of Diagnostic Radiology, University of Maryland Medical Center, Baltimore Maryland, USA \\ "Corresponding author: Seung Min Yoo, Department of Diagnostic Radiology, CHA University College of Medicine, Bundang, Korea. Tel: +82-317805423, Fax: +82-317804800, \\ E-mail: smyoo68@hanmail.net
}

Received 2016 May 26; Revised 2016 July 28; Accepted 2016 August 12.

\begin{abstract}
Diagnosis of cardiac tamponade has been mainly based on clinical features and echocardiography. However, CT may be an initial tool for the evaluation of cardiac tamponade because cutting edge CT is increasingly being installed in the emergency room, and presentation of cardiac tamponade can be nonspecific. Importantly, CT has better spatial resolution compared with echocardiography. Thus, identification of the etiology of cardiac tamponade is possible on CT. We report a case of cardiac tamponade demonstrating direct leakage of contrast material from the right ventricle into the pericardial space caused by central venous catheter injury diagnosed on CT.
\end{abstract}

Keywords: Computerized Tomography, Cardiac Tamponade, Central Venous Catheterization

\section{Introduction}

Acute cardiac tamponade is a life-threatening condition caused by a variety of etiologies including blunt trauma, stab wounds, Stanford type A aortic dissection, or cardiac rupture as a complication of acute myocardial infarction or central venous catheter injury (1-6). Cardiac tamponade is accompanied by hemodynamic instability such that computed tomography (CT) is relatively contraindicated. Thus, the diagnosis of cardiac tamponade is based mainly on clinical features (i.e., the classic triad of hypotension, neck vein distension, and muffled heart sounds) and bedside echocardiography. However, at times CT may be an initial tool for evaluation of cardiac tamponade because state-of-the-art CT scanners are increasingly being installed within the emergency department (ED), and the presentation of cardiac tamponade is often nonspecific. Importantly, CT has better spatial resolution and no limitation of viewing windows compared with echocardiography. Thus, precise identification of the etiology of cardiac tamponade and localization of the site of rupture may be possible on CT, assisting the cardiac surgeon in establishing an appropriate surgical plan. Notably, direct visualization of leakage of contrast material from a cardiac chamber into the pericardial space causing cardiac tam- ponade on CT has not been reported in the literature (1$6)$. We report a case of cardiac tamponade caused by central venous catheter injury, demonstrating direct leakage of contrast material from the right ventricle into the pericardial space on CT.

\section{Case Presentation}

A 75-year-old woman presented to the ED with sudden dyspnea and loss of consciousness during hemodialysis. The patient had no history relevant to cardiac tamponade other than end stage renal disease due to hypertension. Because an arteriovenous fistula in her left arm did not mature fully, hemodialysis had been performed through a right subclavian vein catheter for a year.

Blood pressure was $163 / 68 \mathrm{mmHg}$ at the time of initiation of hemodialysis, but the blood pressure dropped suddenly to $63 / 45 \mathrm{mmHg}$ two hours into the hemodialysis session. On chest radiography performed in the ED, the tip of 14.5 Fr central venous catheter (Hemostar@ Long-term Homodialysis Catheter, BARD Access Systems, Inc., Salt Lake City, USA) projected between the right atrium and ventricle (Figure $1 \mathrm{~A}$ ). As the primary concern of the ED physician was massive pulmonary embolism, a pulmonary CT angiography was performed. The study showed severe 

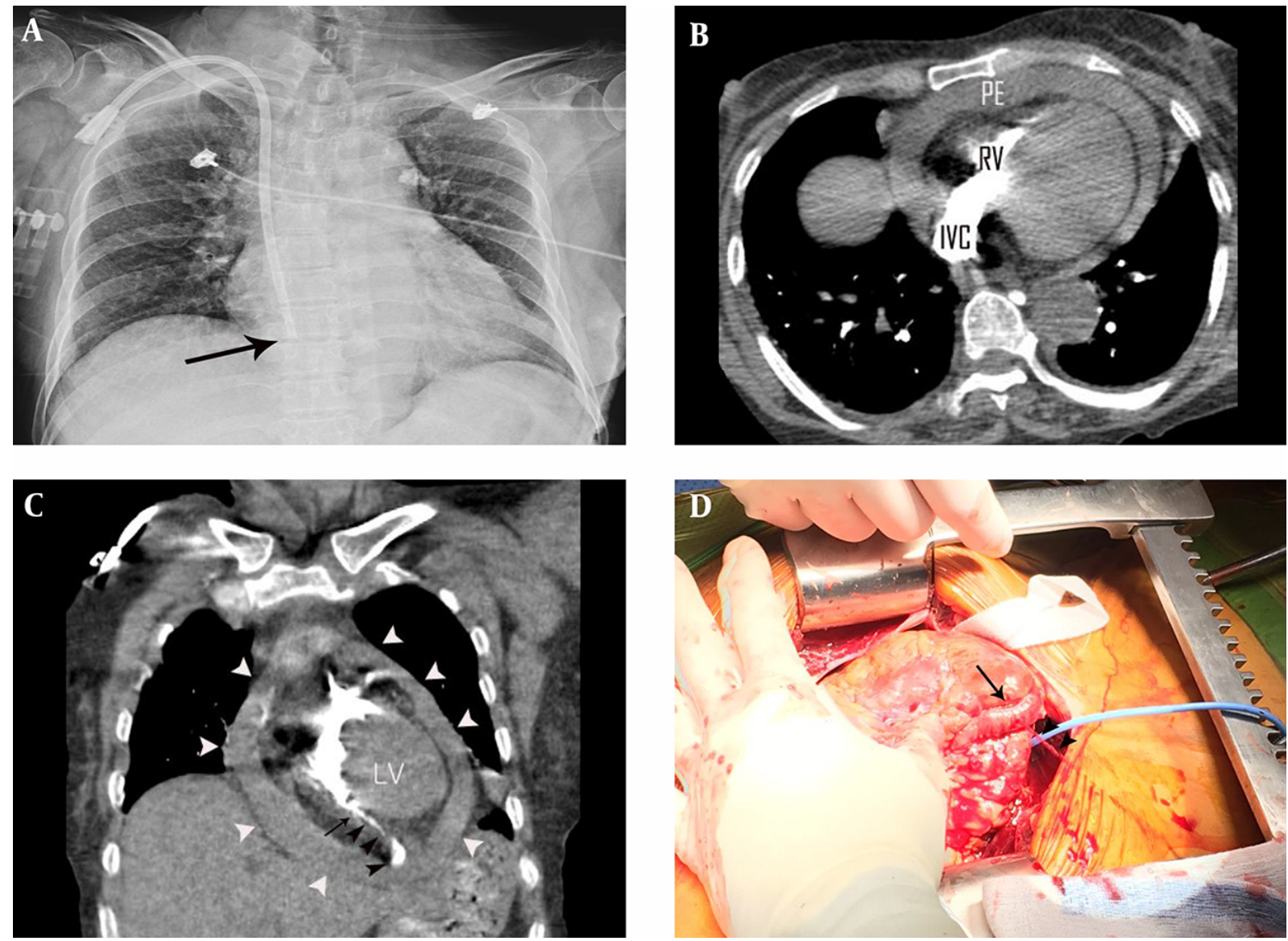

Figure 1. A 75-year-old woman with right ventricular rupture caused by central venous catheter injury. A, Tip of central venous catheter (arrow) is located at the junction of the right atrium and ventricle on a chest radiograph performed in the emergency department. B. A large amount of hemopericardium (PE) compressing the right ventricle (RV) is noted on an axial CT image at the level of the left ventricle. Note marked dilatation of the inferior vena cava (IVC) in contrast to the size of the right ventricle. This constellation of CT findings is typical of cardiac tamponade. C, Direct leakage of contrast materials from the right ventricle into the pericardial space is noted on a coronal reformatted image. Note the site of rupture at the right ventricle (arrow), leakage of contrast materials (black arrowheads), and large amount of hemopericardium (white arrowheads) severely compressing the right ventricle. D, On emergent operation, a 3 - $4 \mathrm{~mm}$ linear defect (arrow) with active bleeding (arrowheads) is noted in the right ventricular free wall.

compression of the right ventricle and atrium by a large hemopericardium and distension of the inferior vena cava, consistent with cardiac tamponade (Figure 1B and 1C). Direct leakage of contrast material (Figure 1C) from the free wall of the right ventricle into the pericardial space was demonstrated on coronal reformatted CT image. A diagnosis of right ventricular rupture caused by central venous catheter injury was made, and emergency surgery was recommended based on CT findings. Impaction of the central venous catheter tip within the right ventricular free wall was demonstrated on the intra-operative echocardiography, suggesting central venous catheter injury as the etiology of cardiac tamponade. At surgery, a 3-4 mm linear tear with active bleeding was found on the right ventricular free wall (Figure 1D), and direct closure of the defect was performed.

\section{Discussion}

Right ventricular rupture caused by central venous catheter injury is extremely rare $(4,5)$. It is known that the tip of the central venous catheter can be moved by changes in neck motion, respiration, or cardiac rhythm (4). Thus, placement of the tip of the central venous catheter in the superior vena cava is optimal to avoid serious complications. Although there are limitations due to a selected set of circumstances (i.e., infants and older catheters), one study reported that the incidence of cardiac tamponade occurring $\geq 7$ days after insertion of a central venous catheter was $18 \%$ (7). On further investigation, the central venous catheter in this patient had been located in the 
right cardiac chamber for approximately one year, leading to an increased risk of cardiac tamponade. Typical CT findings of cardiac tamponade were demonstrated in the present case (i.e., large hemopericardium compressing the right atrium and ventricle and marked dilatation of the inferior vena cava). A previous study has shown that compression of the right ventricle is highly specific for the diagnosis of cardiac tamponade (3). However, there is no prior report demonstrating direct leakage of contrast material from a cardiac chamber to the pericardial space on CT as a finding of cardiac tamponade. Detection of leakage of contrast material into the pericardial space on CT in the present case may have been aided by two factors. First, the size of the defect in the right ventricle by central venous catheter injury may not have been large enough to cause sudden death, thus allowing sufficient time for identification on CT. The second potential reason is that the high injection rate of contrast material in the right ventricle on pulmonary CT angiography may have facilitated detection of leakage into the pericardium. It should be stressed that if only axial CT images are reviewed, the leakage of contrast materials from the right ventricle into the pericardial space may be misinterpreted as an artifact caused by cardiac motion and therefore overlooked. Thus, thorough evaluation of coronal and sagittal CT reformatted images is mandatory in the evaluation of cardiac tamponade.

In summary, the location of the tip of a central venous catheter should be carefully evaluated to avoid the risk of rupture of the right atrium or ventricle. Moreover, ED physicians and radiologists should assess for leakage of contrast material from cardiac chambers into the pericardial space on CT in patients with hemopericardium and an indwelling central venous catheter.

\section{Acknowledgments}

The authors declare that they have no fund or conflict of interest.

\section{Footnotes}

Authors' Contributions: None.

Financial Disclosure: None.

Funding/Support: None.

\section{References}

1. Kim MH, Lee DJ, Kim MC. Bilateral hydrothorax and cardiac tamponade after right subclavian vein catheterization -A case report. Korean J Anesthesiol. 2010;59 Suppl:S211-7. doi:10.4097/kjae.2010.59.S.S211. [PubMed: 21286444].

2. Nichols J, Berger N, Joseph P, Datta D. Subacute right ventricle perforation by pacemaker lead presenting with left hemothorax and shock. Case Rep Cardiol. 2015;2015:983930. doi: 10.1155/2015/983930. [PubMed: 25785204].

3. Spodick DH. Acute cardiac tamponade. N Engl J Med. 2003;349(7):68490. doi: 10.1056/NEJMra022643. [PubMed: 12917306].

4. Ellis LM, Vogel SB, Copeland EM. Central venous catheter vascular erosions. Diagnosis and clinical course. Ann Surg. 1989;209(4):475-8. [PubMed: 2930292].

5. Greenall MJ, Blewitt RW, McMahon MJ. Cardiac tamponade and central venous catheters. Br Med J. 1975;2(5971):595-7. [PubMed: 1131628].

6. Schiavone WA. Cardiac tamponade: 12 pearls in diagnosis and management. Cleve Clin J Med. 2013;80(2):109-16. doi: 10.3949/ccjm.80a.12052. [PubMed: 23376916].

7. Giacoia GP. Cardiac tamponade and hydrothorax as complications of central venous parenteral nutrition in infants. JPEN J Parenter Enteral Nutr. 1991;15(1):110-3. [PubMed: 1901100]. 